\title{
ON THE EVOLUTION OF DOMINANCE
}

\author{
O. MAYO \\ Department of Genetics, University of Adelaide, South Australia
}

Received 27.i.66

\section{INTRODUCTION}

Fisher's reasons for suggesting the evolution of dominance have been set out very clearly (Fisher, 1958, pp. 75-76):

" Examination of the incidence of dominance in mutations observed to occur, and of other genes which must be regarded as mutants, shows that in the majority of cases the wild gene is dominant to the mutant genes, while in a minority of cases dominance is incomplete. Different mutations of the same wild genes show mutually on the other hand a regular absence of dominance. If the substitution of mutant for primitive genes has played any part in evolution, these observations require that the wild allelomorphs must become dominant to their unsuccessful competitors. The incidence of heterozygotes of each mutant among the ancestry of the wild population is, if we may rely upon observed mutation rates to be of the right order of magnitude, sufficient to account for the evolution of dominance by the selection of modifying factors. This process is extremely slow, since the proportion of the population effectively exposed to selection is about I in Io,000 or I00,000."

Because of the nature of this argument, it is clear that there can be two kinds of objection to the theory: first those which criticise the efficacy of the selection in favour of modifiers, or which criticse the methods used for the evaluation of these selective forces (Wright, I 929 $a, b$; Haldane, I932; Crosby, I963; Ewens, I965 $a, b$ ); and secondly those criticisms, based on studies of gene action, which suggest that dominance may in general be an intrinsic property of gene function, not to be expected of defective (mutant) alleles (Wright, 1929a, $b$, I 934, etc.). We are here concerned mainly with the first kind of criticism, and do not attempt to answer the question: " which of these factors is more important in the evolution of dominance of the wild type allele, selection for modifiers, or selection for the most potent alleles "? (Wagner and Mitchell, 1955.)

This paper also describes some computer simulation studies of rapidly changing populations where dominance may evolve perhaps more quickly than otherwise (Haldane, 1956; Parsons and Bodmer, 196r). The results of these experiments are discussed in relation to the criticisms that have been made of Fisher's theory.

\section{THE WORK OF CROSBY}

Crosby (1963) describes his paper as a strong criticism of Fisher's theory. For example, of Fisher's (1935, 1938) experiments on poultry 
to test the effect of a changed genetic background on the expression of dominant mutant genes, Crosby writes, "I find the results of his experiments very unconvincing". However, he only discusses one of the experiments in any detail, and may have misinterpreted this one, since Fisher regarded the apparent dominance of Crest as being due to suppression by modifying factors of the deleterious effect of the gene. That is to say, Fisher held that when the birds were originally being selected for Crest, this would have been carried out mainly on heterozygotes, so that the effect of modifiers on the homozygous mutant should not have been important. This is perhaps contrary to Crosby's suggestion, that the deleterious effect of Crest does not appear in any genotype in the domestic breed because of selection against its expression in the homozygote, so that "the reappearance of this suppressed character after backcrossing accounts for the differentiation of the heterozygote".

Whichever view is adopted, modifiers which suppressed the deleterious effect of the gene for Crest in the homozygote, as well as enhancing the effect on the crest itself in the heterozygote, would be selected since the breeder would desire to develop a true-breeding stock.

Crosby also discusses a numerical example, and suggests that computer simulation may be used to study the course of dominance modification with time. This suggestion has been followed in the present paper; the simulated " experiments" are described in section 4 below.

The numerical example considered by Crosby consists of a population of size I,000,000, with two loci segregating, $A, a$ and $M, m$. Neither locus exhibits dominance in respect of fitness. There is mutation from $A$ to $a$ at the rate of ${ }_{10} 0^{-6}$ per gene per generation, and the genotypes have selective values as shown:

\begin{tabular}{lcll} 
& $A A$ & \multicolumn{1}{c}{$A a$} & $a a$ \\
$M M$ & $\mathrm{I}$ & $\mathrm{I}$ & 0.5 \\
$M m$ & $\mathrm{I}$ & 0.75 & 0.5 \\
$\mathrm{~mm}$ & $\mathrm{I}$ & 0.5 & 0.5
\end{tabular}

When selection balances mutation, there are $8 A a$ in the population, and selection for $M$ can only operate on these. Selection increases the number of $M$ genes by one in the first generation. Crosby appears to regard such selection as ineffective, mainly because of sampling effects. He does not discuss the point, agreed on by Fisher and Wright, that the intensity of selection in favour of $M$ will increase with $q_{M}$, the frequency of $M$, or the allied fact that " evolution of dominance proceeds with increasing speed as dominance becomes more complete " (Fisher, 1929), where dominance need not be induced simply by a single modifier.

Further, Crosby does not discuss the time required for dominance to evolve, except to say that it is difficult to produce " a model which would show a result in a reasonable time" by computer simulation. 
While it is not clear whether this is biological time or computer time, there is no suggestion that 100,000 generations or more were considered. This was near the order of magnitude of time proposed by Fisher for dominance to evolve.

\section{THE WORK OF EWENS}

Ewens $(1965 a, b)$ states that he has found an error in Fisher's work on a point which Fisher regards as of "essential importance" to his theory. In considering Ewens' criticisms, it is first necessary to see what was regarded as essential: Fisher (1928) showed that "the fraction of the ancestry of future generations ascribable to heterozygotes " for the mutant allele "decreases very rapidly as $v \rightarrow 0$, and also increases very rapidly as $v \rightarrow I$. The early states in the improvement of the heterozygote will necessarily be retarded very much more severely than the later states, which may be expected to be passed through quite rapidly in comparison ". (Here, $v$ is the selective value of the heterozygote.)

Wright (I929a), in criticising Fisher's theory, introduced the particular model of dominance of an allele produced by a dominant allele $M$ at another locus, and arrived at a result suggesting that the intensity of selection for $M$ would decrease as it approached fixation, i.e. as $q_{M} \rightarrow \mathrm{I}$. Fisher (1 929) in replying to this, pointed out that for Wright's model, the intensity of selection would, in fact, increase without limit as the modifying allele approached fixation - a correction with which Wright $\left(19^{29} b\right)$ agreed. After his very brief reference to Wright's model, Fisher (1949) wrote "I do not in the least wish to dispute that the selective intensity will be proportional to, and generally of the order of, the mutation rate, though the fact that the evolution of dominance proceeds with increasing speed as dominance becomes more complete is an essential point stressed in my original note". In the "original note" (Fisher, 1928), the theory of the evolution of dominance was developed without reference to the particular model or formula which was later introduced by Wright. Ewens (1965a) has written "The evolution of dominance analyses of Fisher and Wright were carried out in terms of the frequency $q_{M}$ of $M$ ", and again (Ewens, 1965b) "it is interesting to note that Fisher originally developed it (the theory of the evolution of dominance) using an incorrect formula and that had he been aware of the correct formula he would not have advanced his theory at all ". We therefore examine Ewens' mathematical approach in some detail.

Ewens supposes that we have two loci, $A, a$ and $M, m$ with selective values and genotypic frequencies as shown:

\begin{tabular}{|c|c|c|c|c|c|c|c|}
\hline \multicolumn{4}{|c|}{ Selective Values } & \multicolumn{4}{|c|}{ Genotype Frequencies } \\
\hline & $A A$ & $A a$ & $a a$ & & $A A$ & $A a$ & $a a$ \\
\hline$M M$ & I & I & I $-S$ & $M M$ & $c_{1}^{2}$ & $2 c_{1} c_{2}$ & $c_{2}^{2}$ \\
\hline$M m$ & I & I & I $-S$ & $M m$ & $2 c_{1} c_{3}$ & $2 c_{1} c_{4}+2 c_{2} c_{3}$ & $2 c_{2} c_{4}$ \\
\hline $\mathrm{mm}$ & I & I $-s h$ & I $-S$ & $\mathrm{~mm}$ & $\mathrm{c}_{3}^{2}$ & $2 c_{3} c_{4}$ & \\
\hline
\end{tabular}


Here, following Moran (1964), the genotypic frequencies at one locus are not necessarily distributed at random with respect to those at the other locus, and $c_{1}, c_{2}, c_{3}$ and $c_{4}$ are the frequencies of the gametes $A M$, $a M, A m$ and $a m$, respectively. $A$ is not dominant to $a$, but $M$ is to $m$ in its effect on the heterozygote, $A a$.

If $u$ is the mutation rate from $A$ to $a$, the equilibrium frequency of $A$ is approximately $\mathrm{I}-\frac{u}{s h}$, in the absence of $M$.

Following Moran, Ewens shows that the change in $q_{M}$, the frequency of $M$, per generation due to selection is approximately given by

$$
\Delta q_{M}=q_{M}\left(2 s h c_{3} c_{4}\right)+o\left(h^{2}\right) .
$$

It is clear that, under random mating with no selection, if $q_{A}$ is the frequency of the gene $A$, then

$$
\begin{aligned}
c_{3} & =q_{A}\left(\mathrm{I}-q_{M}\right) \\
c_{4} & =\left(\mathrm{I}-q_{A}\right)\left(\mathrm{I}-q_{M}\right) .
\end{aligned}
$$

(Substitution from (2) in (I) gives (3) below.) Moran showed that these relationships are invalid where selection is acting, but that for small $s$, they are reasonable approximations. If these relationships are to be used, Moran's analysis is not required, as the same result is seen very simply as follows.

We have selective values as shown above and the following frequencies for the nine possible genotypes:

\section{$A A$}

$M M \quad q_{A}^{2} q_{M}^{2}$

$M m \quad 2 q_{A}^{2} q_{M}\left(\mathrm{I}-q_{M}\right)$

$m m \quad q_{A}^{2}\left(\mathrm{I}-q_{M}\right)^{2}$
Aa

$$
\begin{aligned}
& 2 q_{A}\left(\mathrm{I}-q_{A}\right) q_{M}^{2} \\
& 4 q_{A}\left(\mathrm{I}-q_{A}\right) q_{M}\left(\mathrm{I}-q_{M}\right)
\end{aligned}
$$$$
2 q_{A}\left(\mathrm{I}-q_{A}\right)\left(\mathrm{I}-q_{M}\right)^{2}
$$

aa

$$
\left(\mathrm{I}-q_{A}\right)^{2} q_{M}^{2}
$$$$
2\left(\mathrm{I}-q_{A}\right)^{2} q_{M}\left(\mathrm{I}-q_{M}\right)
$$$$
\left(\mathrm{I}-q_{A}\right)^{2}\left(\mathrm{I}-q_{M}\right)^{2}
$$

It follows that

$$
\text { mean selective value }(\bar{S})=\mathrm{I}-2 q_{A}\left(\mathrm{I}-q_{A}\right) q_{M}^{2} s h-q_{A}^{2} s .
$$

Hence, the frequency of $M$ in the next generation is $q_{M}^{1}$, given by

$$
q_{M}^{1}=\frac{q_{A}^{2} q_{M}+2 q_{A}\left(\mathrm{I}-q_{A}\right) q_{M}+\left(\mathrm{I}-q_{A}\right)^{2} q_{M}(\mathrm{I}-s)}{\bar{S}} .
$$

Thus,

$$
\begin{aligned}
\Delta q_{M} & =q_{M}^{3}-q_{M} \\
& =\frac{2 q_{A}\left(\mathbf{1}-q_{A}\right) q_{M}\left(\mathbf{1}-q_{M}\right)^{2} s h}{\bar{S}}
\end{aligned}
$$

i.e. for small $s$,

$$
\Delta q_{M}=2 q_{A}\left(\mathrm{I}-q_{A}\right) q_{M}\left(\mathrm{I}-q_{M}\right)^{2} s h .
$$

As mentioned above, in his earlier analysis, to obtain a simpler 
expression for $\Delta q_{M}$, Ewens used $q_{A}^{(1)}=\mathrm{I}-(u / s h)$ for $q_{A}$, and approximating in ( $\mathrm{I}$ ) from

obtained

$$
\begin{aligned}
c_{3} & =\mathrm{I}-q_{M}+\mathrm{O}(u) \\
c_{4} & =\frac{u\left(\mathrm{I}-q_{M}\right)}{s h}
\end{aligned}
$$

$$
q_{M}=2 u q_{M}\left(\mathrm{I}-q_{M}\right)^{2}
$$

which is essentially the value which Wright (I929b) accepted as incorrect. However, in his second paper, Ewens $\left(\mathrm{I}_{96} b\right)$ recognises that both $q_{A}^{(1)}$ and $q_{A}^{(2)}=\mathrm{I}-\sqrt{ } u / s$, the value for complete dominance of $A$ over $a$, are incorrect, since $q_{A}$ is changing from $q_{A}^{(1)}$ to $q_{A}^{(2)}$. As Ewens indicates, derivation of an exact expression for $q_{A}$ in this range " does not appear to be simple ", and an approximate method is required.

Fisher appears to have considered the value of $q_{A}$ obtained from the limit $\Delta q_{A} \rightarrow 0$, which is, approximately

$$
q_{A}^{(3)}=\mathrm{I}-\frac{u}{\operatorname{sh}\left(\mathrm{I}-q_{M}\right)^{2}} .
$$

For such values of $q_{M}$ as are less than or equal to I $-\left(\frac{u}{h^{2} S}\right)^{\frac{1}{4}}$,

$$
q_{A}^{(1)} \geqslant q_{A}^{(3)} \geqslant q_{A}^{(2)},
$$

so that $q_{A}^{(3)}$, which Ewens considers to be invalid because $\Delta q_{A}=0$ at $q_{M}=\mathrm{I}$ where (6) does not hold, is a reasonable value to adopt while selection is occurring.

If we now substitute from (6) in (3), we obtain, approximately,

$$
\Delta q_{M}=2 u q_{M}
$$

which was essentially obtained by Fisher (1929) and accepted by Wright $($ I $929 b)$.

Another point considered by Ewens is the magnitude of scale effects of selective advantage. He puts forward an example, which, he suggests, shows that Fisher's (I929) assumption that " a small selective intensity of say I/50,000 the magnitude of a larger one will produce the same effect in 50,00o times the time " is in error. If the effects of the modifying genes are small and additive, this assumption should hold, since Fisher, considering selective intensities in terms of their action on $z=\log (q / \mathrm{I}-q)$, showed (1930, I958) that "the effects of selection in modifying the gene frequencies are, on the logarithmic scale, exhibited with the utmost simplicity, namely by changes of position with velocities that are uniform and proportional only to the intensity of selection". Thus, Ewens' example, which follows, is perhaps not really relevant to Fisher's theory.

Ewens gives the following results for " a diploid population of size ${ }^{10} 0^{6 "}$ with two equally frequent alleles, where $a$ is the selective advantage 
of the superior allele and $P$ is the probability of the fixation of this allele:

$\begin{array}{cccc}a & \Delta a & P & \Delta P \\ 0 & & 0.5 & 0.38 \mathrm{I} \\ 2 \cdot 10^{-6} & 2 \cdot 10^{-6} & 0.88 \mathrm{I} & \\ 8 \cdot 10^{-6} & & 0.99966 & \\ \mathrm{IO}^{-5} & 2 \cdot 10^{-6} & 0.00029\end{array}$
viz.

Ewens apparently used a formula given by Moran (I962, p. I I 8 )

$$
P=\frac{\mathrm{I}-e^{-2 k} o^{a}}{\mathrm{I}-e^{-2 n a}}
$$

where $k_{o}$ is the initial number of advantageous alleles, and $n$ is twice the diploid population size. However, it is not certain that he did, since the values shown above require $k_{\circ}=500,000$ and $n=\mathrm{I}, 000,000$, which describe a diploid population of size 500,000. The values obtained from (8) for the conditions given by Ewens are:

$\begin{array}{cccc}a & \Delta a & P & \Delta P \\ 0 & & 0 \cdot 5 & 0 \cdot 4820 \mathrm{I} \\ 2 \cdot 10^{-6} & 2 \cdot 10^{-6} & 0 \cdot 9820 \mathrm{I} & \\ 8 \cdot 10^{-6} & & \mathrm{I} \cdot 00000 & \\ 10^{-5} & 2 \cdot 10^{-6} & 1 \cdot 00000 & 0 \cdot 00000\end{array}$

Using Ewens' values, or those obtained here from (8), it is clear that the two equal values of $\Delta a$ give very different values of $\Delta P$. This, Ewens considers, shows how the effect may not be proportional to the cause. It is not clear why a change from a random event (fixation in the absence of selection) to a partially determinate event (fixation in the presence of selection) should be comparable to a simple change in the latter event. Further, it should be noted that Moran's expression (8) refers to the probability of ultimate fixation, i.e. in an indefinitely long time, and not to the rate of approach to fixation, which is the variable relevant to dominance modification.

\section{SIMULATION OF SIMPLE MODIFICATION OF DOMINANCE}

Haldane ( 1956 ) and Parsons and Bodmer (I96I) have considered the possibility of dominance modification in the case where a new, advantageous mutant gene $A$ appears in a population of $a a$ individuals. If $A$ is not dominant to $a$, while $A$ is being fixed, there will be a large number of heterozygotes on which selection for dominance of $A$ can act. Haldane has concluded that "it is improbable that the frequency of a modifier would increase as much as 20 times, though it might 
increase ro times.... The process would be slower if the modifier were not rare or not dominant". Parsons and Bodmer, considering the possibility of the evolution of heterozygote advantage (overdominance), feel that the frequency could rise as much as roo times, though with slightly different initial conditions. Since in this case heterozygotes are much more frequent, selection for modifiers may be expected to be effective in hundreds, rather than hundreds of thousands, of generations. Thus, this case should be more amenable to simulation with a digital computer than Fisher's original suggestion. Crosby has indeed considered the simplest case.

In the experiments carried out by the author, the populations were of diploid organisms, mating was at random, generations were non over-lapping and selection took place after the formation of the zygote, to determine whether a new individual would be an adult in the next generation. For details of the mechanics of simulation of this kind see, e.g. Gill ( $(965)$. The computer used was a Control Data Corporation 3600 .

Population size was 50o. Several numbers of modifier loci ( 1,2 and 10) were used. All loci segregated independently of each other. The experimental results are described below. Mean values shown in the tables are from at least four trials of each experiment. A mutation rate from $A$ to $a$ of ${ }_{10}^{-6}$ per gene per generation was used.

\section{(i) A single locus undergoing selection, with segregation at one modifying locus}

Initial frequencies of $a a$ individuals were as follows:

\begin{tabular}{lclllclcc}
\multicolumn{3}{r}{ Experiments $(a)$ and $(c)$} & \multicolumn{4}{c}{ Experiment $(b)$} \\
& $A A$ & $A a$ & $a a$ & & $A A$ & $A a$ & $a a$ \\
$M M$ & 0 & 0.005 & 0.245 & $M M$ & 0 & 0 & 0.02 \\
$M m$ & 0 & 0.01 & 0.49 & $M m$ & 0 & 0 & 0.25 \\
$m m$ & 0 & 0.0005 & 0.245 & $m m$ & 0 & 0.05 & 0.68
\end{tabular}

Selective values are shown in table I. In experiments $(a)$ and (b), $M$ and $m$ are selectively neutral, apart from their effects on $A a$. In experiment $(c)$, they are maintained in a balanced polymorphism in the absence of $A$. Results are shown in table 2.

(ii) A single locus undergoing selection with segregation at two modifying loci

Initial genotypic frequencies in these experiments were as follows:

Experiments $(a)$ and $(b)$

$\begin{array}{lccl} & M_{1} M_{1} & M_{1} m_{1} & m_{1} m_{1} \\ M_{2} M_{2} & 0.25 & 0 & 0 \\ M_{2} m_{2} & 0 & 0.5 & 0 \\ m_{2} m_{2} & \circ & 0 & 0.23 \\ & 2 \mathrm{~K} & & \end{array}$

Experiments $(c)$ and $(d)$ $M_{1} M_{1} \quad M_{1} m_{1} \quad m_{1} m_{1}$ $0.023 \quad 0 \quad 0$ $\begin{array}{lll}0 & 0.255 \quad 0\end{array}$ $\begin{array}{lll}0 & 0 & 0.702\end{array}$ 
The frequency of $A A$ was zero, and that of $A a m_{1} m_{1} m_{2} m_{2}$ was 0.02 . (The alleles at the two modifying loci are $M_{1}, m_{1}$ and $M_{2}, m_{2}$.) The selective value was I for all $A A$ individuals. The selective values of the heterozygotes $(A a)$ are shown in table 3 . In experiments $(a)$ and $(b)$, the value for all $a a$ individuals was $0 \cdot 8$, and in experiments $(c)$ and $(d)$, o.6. Results are shown in table 4 .

TABLE I

Selective values

\begin{tabular}{|c|c|c|c|c|}
\hline \multirow[b]{2}{*}{$\begin{array}{c}\text { Experiment } \\
(a)\end{array}$} & & $A A$ & $A a$ & $a a$ \\
\hline & $\begin{array}{l}M M \\
M m \\
m m\end{array}$ & $\begin{array}{l}1 \\
1 \\
1\end{array}$ & $\begin{array}{l}1 \\
0.95 \\
0.9\end{array}$ & $\begin{array}{l}0.8 \\
0.8 \\
0.8\end{array}$ \\
\hline \multirow{3}{*}{$\begin{array}{l}\text { Experiment } \\
(\text { b })\end{array}$} & & $\mathrm{AA}$ & $\mathrm{Aa}$ & $\mathbf{a a}$ \\
\hline & $\begin{array}{l}M M \\
M m \\
m m\end{array}$ & $\begin{array}{l}\text { I } \\
\text { I } \\
\text { I }\end{array}$ & $\begin{array}{l}1 \\
0.9 \\
0.8\end{array}$ & $\begin{array}{l}0.6 \\
0.6 \\
0.6\end{array}$ \\
\hline & & $A A$ & $A a$ & $a a$ \\
\hline $\begin{array}{c}\text { Experiment } \\
(c)\end{array}$ & $\begin{array}{l}M M \\
M m \\
m m\end{array}$ & $\begin{array}{l}0.9 \\
1 \\
0.9\end{array}$ & $\begin{array}{l}0.9 \\
0.95 \\
0.81\end{array}$ & $\begin{array}{l}0.7^{2} \\
0.8 \\
0.72\end{array}$ \\
\hline
\end{tabular}

TABLE 2

Mean time to, and frequency of $M$ at, fixation of $A$

\begin{tabular}{|c|c|c|}
\hline Experiment & $\begin{array}{l}\text { Number of } \\
\text { generations }\end{array}$ & $q_{M}$ \\
\hline $\begin{array}{l}(a) \\
(b) \\
(c)\end{array}$ & $\begin{array}{r}131 \pm 1 \cdot 21 \\
59 \cdot 2 \pm 2 \cdot 82 \\
112 \cdot 25 \pm 6 \cdot 24\end{array}$ & $\begin{array}{l}0.754 \pm 0.056 \\
0.348 \pm 0.047 \\
0.512 \pm 0.063\end{array}$ \\
\hline
\end{tabular}

(iii) A single locus undergoing selection with segregation at ten modifying loci

The selective values were arranged so that each advantageous modifying allele, at whichever of the ten loci it was, had the same effect; and these effects (on the heterozygote) were simply additive. Thus, a heterozygote, $A a$, carrying advantageous modifying alleles at sufficient of the twenty sites to induce complete dominance would resemble the 
advantageous homozygote, $A A$; a heterozygote carrying the alternative allele at all twenty sites would resemble the heterozygote in the absence of dominance modifiers; and the other heterozygotes would be intermediate in selective value, depending on how many advantageous modifying alleles they carried. Five experiments were carried out: dominance of $A$ over $a$ induced $(a)$ by 20 advantageous alleles, $(b)$ by

\section{TABLE 3}

Selective values of the heterozygotes (Aa)

\begin{tabular}{|c|c|c|c|c|}
\hline \multirow[b]{2}{*}{$\underset{(a)}{\text { Experiment }}$} & & $M_{1} M_{1}$ & $M_{1} m_{1}$ & $m_{1} m_{1}$ \\
\hline & $\begin{array}{l}M_{2} M_{2} \\
M_{2} m_{2} \\
m_{2} m_{2}\end{array}$ & $\begin{array}{l}I \\
0.975 \\
0.95\end{array}$ & $\begin{array}{l}0.975 \\
0.95 \\
0.925\end{array}$ & $\begin{array}{l}0 \cdot 95 \\
o \cdot 925 \\
0 \cdot 9\end{array}$ \\
\hline \multirow{3}{*}{$\begin{array}{l}\text { Experiments } \\
(b) \text { and }(c)\end{array}$} & & $M_{1} M_{1}$ & $M_{1} m_{1}$ & $m_{1} m_{1}$ \\
\hline & $\begin{array}{l}M_{2} M_{2} \\
M_{2} m_{2} \\
m_{2} m_{2}\end{array}$ & $\begin{array}{l}1 \\
0.95 \\
0.9\end{array}$ & $\begin{array}{l}0 \cdot 95 \\
0 \cdot 9 \\
0 \cdot 85\end{array}$ & $\begin{array}{l}0.9 \\
0.85 \\
0.8\end{array}$ \\
\hline & & $M_{1} M_{1}$ & $M_{1} m_{1}$ & $m_{1} m_{1}$ \\
\hline$\underset{(d)}{\text { Experiment }}$ & $\begin{array}{l}M_{2} M_{2} \\
M_{2} m_{2} \\
m_{2} m_{2}\end{array}$ & $\begin{array}{l}\text { I } \\
\text { I } \\
\text { O.933 }\end{array}$ & $\begin{array}{l}1 \\
0.933 \\
0.867\end{array}$ & $\begin{array}{l}0.933 \\
0.867 \\
0.8\end{array}$ \\
\hline
\end{tabular}

TABLE 4

Mean time to, and mean modifier frequency at, fixation of $A$

\begin{tabular}{|c|c|c|}
\hline Experiment & $\begin{array}{c}\text { Number of } \\
\text { generations }\end{array}$ & $\bar{q}_{M}$ \\
\hline & & \\
\hline$(a)$ & $129 \cdot 75 \pm 13 \cdot 24$ & $0 \cdot 697 \pm 0 \cdot 061$ \\
$(b)$ & $67 \cdot 8 \pm 6 \cdot 55$ & $0 \cdot 626 \pm 0 \cdot 037$ \\
$(c)$ & $43 \cdot 0 \pm 5 \cdot 01$ \\
$(d)$ & $5 \cdot \cdot 2 \pm 4 \cdot 87$ & $0 \cdot 229 \pm 0 \cdot 027$ \\
\hline
\end{tabular}

15 or more advantageous alleles, $(c)$ by Io or more advantageous alleles, $(d)$ by 7 or more advantageous alleles, and $(e)$ by 4 or more advantageous alleles. This situation is shown in table 5. $s=0.2$ was used in all experiments. The selective value of all $A A$ genotypes was $\mathrm{r}$, and that for all $a a$ genotypes was $\mathrm{I}-2 s$.

Initially, $q_{A}$ was 0.05 , and the frequency of $A A$ was zero. Initial 
O. MAYO

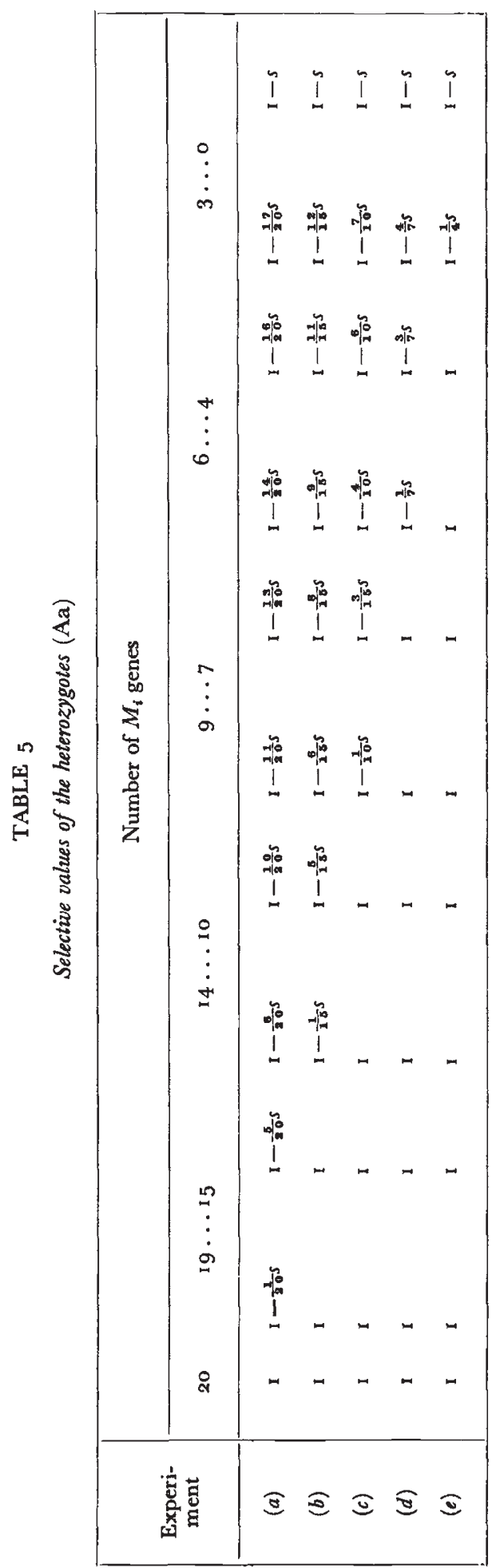


frequencies of the $M_{i}, i=\mathrm{I}, 2 \ldots$ Io, are shown in table 6 . Genotypes for the ten modifying loci were assigned at random to each individual, except that the $A a$ individuals were $m_{i} m_{i}, i=\mathrm{I}, 2 \ldots$ Io.

Results are shown in table 6. $q_{M}$ is the overall mean for all $M_{i}$, $i=\mathrm{I}, \ldots \mathrm{IO}$.

\section{TABLE 6}

Mean time to, and mean modifier frequency, $\overline{\mathrm{q}}_{\mathrm{M}}$, at fixation of $A$

\begin{tabular}{|c|c|c|c|}
\hline Experiment & $\begin{array}{c}\text { Initial } \\
q_{M_{i}}\end{array}$ & $\begin{array}{l}\text { Number of } \\
\text { generations }\end{array}$ & $\bar{q}_{M}$ \\
\hline $\begin{array}{l}(a) \\
(a) \\
(b) \\
(b) \\
(c) \\
(c) \\
(d) \\
(c)\end{array}$ & $\begin{array}{l}0.5 \\
0.15 \\
0.5 \\
0.15 \\
0.5 \\
0.15 \\
0.15 \\
0.15\end{array}$ & $\begin{array}{c}57 \cdot 0 \pm 4 \cdot 20 \\
43 \cdot 8 \pm 1 \cdot 74 \\
68 \cdot 8 \pm 2 \cdot 54 \\
42 \cdot 4 \pm 0 \cdot 68 \\
155 \cdot 3 \pm 25 \cdot 11 \\
66 \cdot 2 \pm 6 \cdot \cdot 30 \\
55^{\cdot} \cdot 2 \pm 6 \cdot 10 \\
111 \cdot 2 \pm 23 \cdot 68\end{array}$ & $\begin{array}{l}0.525 \pm 0.017 \\
0.189 \pm 0.021 \\
0.507 \pm 0.016 \\
0.194 \pm 0.009 \\
0.568 \pm 0.020 \\
0.198 \pm 0.013 \\
0.191 \pm 0.013 \\
0.226 \pm 0.018\end{array}$ \\
\hline
\end{tabular}

\section{DISCUSSION AND CONCLUSIONS}

In every experiment carried out, the amount of dominance, as measured by the frequency of the advantageous allele at the modifying locus, or the mean frequency of the advantageous alleles for more than one modifying locus, was increased by the time the advantageous allele at the locus being modified was fixed.

This increase was seen even when the modifying locus was maintained as a balanced polymorphism by quite intense selection. However, the effect was then less marked, and because of the change of selective forces when $A$ is fixed, it is not yet clear how such results will affect the suggestion of Wright ( $\left.19^{2} 9^{b}\right)$ and Crosby that any effect of a modifying locus on dominance would be a fortuitous result of some other kind of selection, i.e. "first-order" selection acting on the modifier. To examine this further, programmes such as those used above will have to be run for many more generations.

The increases in $q_{M}$ were greater than the increases in $q_{M}$ for given selective values. Nevertheless, as can be seen from the experiment with ten modifying loci, modifiers do not need to have large effects to be favoured by selection, even in the very small populations considered here. In particular, the results of those experiments where less than twenty advantageous genes gave dominance suggest that this may be the situation most resembling evolved or evolving dominance in nature. For dominance increased most in the case where fewest of the twenty possible sites had to be occupied by advantageous alleles. Thus, dominance throughout a population should be possible with the population still polymorphic at a considerable number of modifying loci. The rather different situation, where the heterozygote is clearly 
distinguishable from both homozygotes but where the population is polymorphic at a number of modifying loci, has been used in experimental modification of dominance, for example by Ford (1940) in his experiments on modification of the semi-lutea heterozygote in Abraxas grossulariata.

If such dominance as has evolved is indeed determined by the action of a large number of (polygenic) modifiers, the " intuitive " plausibility, suggested by Ewens ( $1965 a$ ), of the decline in the rate of increase of dominance as dominance becomes more complete is not readily apparent, since selection against the less dominant of the heterozygotes becomes more important. Further, much of the discussion of evolution of dominance in terms of the large effect of a single locus would seem to be not particularly relevant to the situation in natural populations.

Even if such discussion is not perfectly relevant, the simplest cases can still shed light on the problem of dominance modification, as this re-examination of criticisms of the original theory may have shown. In particular, it does seem clear that Fisher's essential requirement for dominance to evolve is followed both in the general case (Fisher, I928) and in the example considered by Wright (1929a, b) and Fisher (1929).

\section{SUMMARY}

Recent criticisms of Fisher's theory of the evolution of dominance are discussed in the light of Fisher's original papers. The criticisms considered relate to the efficacy of the selection in favour of modifiers of dominance and to the correctness of the methods used for the evaluation of these selective forces. It is concluded that, in general, Fisher's methods and conclusions appear correct.

Results of computer simulations of dominance modification in rapidly changing populations where dominance may evolve more quickly than otherwise are presented. These support the contention that dominance modification, when it occurs, is produced by many modifiers.

Acknowledgments.-The author wishes to thank Professor J. H. Bennett for many valuable suggestions. He also wishes to thank Professor E. B. Ford, F.R.S., and his colleagues, and a referee, for several helpful criticisms.

\section{REFERENCES}

CROSBY, J. L. 1963. The evolution and nature of dominance. F. Theoret. Biol., 5, 35-5I.

EWENs, W. J. 1965a. A note on Fisher's theory of the evolution of dominance. Ann. Hum. Genet., Lond., 29, 85-88.

EWENs, W. J. r $965 b$. Further notes on the evolution of dominance. Heredity, 2o, 443-450.

FISHER, R. A. 1928. The possible modification of the response of the wild type to recurrent mutation. Amer. Nat., 62, I 1 5-1 26.

Fisher, R. A. 1929. The evolution of dominance; reply to Professor Wright. Amer. Nat., ${ }_{3}, 553-556$. 
FISHER, R. A. 1935. Dominance in poultry. Phil. Trans. Roy. Soc., B, 225, 197-226. FISHER, R. A. 1938. Dominance in poultry. Feathered feet, Rose comb, Internal Pigment and Pile, Proc. Roy. Soc., B, 125, 25-48.

FISHER, R. A. 1930, I958. The Genetical Theory of Natural Selection. Revised 2nd Ed. 1958. New York, Dover.

FORD, E. B. 1940. Genetic research in the Lepidoptera. Ann. Eugen., Lond., Io, $227-252$.

GILL, J. L. 1965. Effects of finite size on selection advance in simulated genetic populations. Aust. F. Biol. Sci., I8, 599-6 I 7 .

haldane, J. B. S. 1932. The Causes of Evolution. Longmans, Green. London.

HALDANE, J. B. s. I956. The theory of selection for melanism in Lepidoptera. Proc. Roy. Soc., B., 145, 303-306.

MORAN, P. A. P. 1962. Statistical Processes of Evolutionary Theory. Oxford, University Press.

Moran, P. A. P. I 964 . On the non-existence of adaptive topographies. Ann. Hum. Genet., Lond., 27, 383-393.

PARSONS, P. A., AND BODMER, W. F. 1961. The evolution of over-dominance: natural selection and heterozygote advantage. Nature, Lond., 190, 7-1 2.

WAgner, R. P., AND mitchelt, H. K. 1955. Genetics and Metabolism. ist Ed. New York, Wiley.

WRIGHT, s. I929a. Fisher's theory of dominance. Amer. Nat., 63, 274-279.

WRIGHT, s. Ig29b. The evolution of dominance. Comment on Dr Fisher's reply. Amer. Nat., 63, 556-56r.

WRight, s. I934. Physiological and evolutionary theories of dominance. Amer. Nat., 68, 24-53. 